\title{
Modelos teóricos e algoritmos para a otimização da alocação de canais em redes móveis sem fio
}

\author{
Bruno Raphael Cardoso Dias ${ }^{1}$ \\ Orientadora: Prof ${ }^{\mathrm{a}}$. Dr ${ }^{\mathrm{a}}$. Rosiane de Freitas Rodrigues ${ }^{1}$ \\ Coorientador: Prof. Dr. Nelson Maculan Filho ${ }^{2}$
}

${ }^{1}$ Instituto de Computação - Universidade Federal do Amazonas (UFAM)

Av. Rodrigo Otávio, 3000 - 69000-000 - Manaus - AM - Brasil

${ }^{2}$ PESC/COPPE - Universidade Federal do Rio de Janeiro (UFRJ)

Caixa Postal 68530 - 21945-970 - Rio de Janeiro - RJ - Brasil

\{bruno.dias, rosiane\}@icomp.ufam.edu.br, maculanecos.ufrj.br

\begin{abstract}
Channel allocation problems are found in the planning of telecommunication networks, where we must assign a channel for outgoing call restrictions respecting separation among transceiver stations, in order to optimize some criterion. In this work, this problem was explored with combinatorial optimization approach, with the proposition of new theoretical models involving graph coloring, scheduling on parallel machines and distance geometry, as well as the definition of exact and approximate algorithmic strategies, involving integer and constraint programming, and heuristics based on local search for solving them. The computational results obtained were competitive, which proved optimality in some cases and obtained better results and better performance in others. In short, the proposed approaches allow the tools of other problems is applied to the planning of mobile wireless networks and present new scientific challenges to the area.
\end{abstract}

Resumo. Problemas de alocação de canais são encontrados no planejamento de redes de telecomunicações, onde deve-se atribuir um canal por chamada efetuada respeitando restrições de separação entre estações transceptoras, de forma a otimizar algum critério. Neste trabalho, o problema foi explorado com enfoque de Otimização Combinatória, com a proposição de novos modelos teóricos envolvendo coloração de grafos, escalonamento em máquinas paralelas e geometria de distâncias, bem como com a definição de estratégias algorítmicas exatas e aproximadas envolvendo programação inteira e por restrições, e heurísticas baseadas em busca local, para a resolução dos mesmos. Os resultados computacionais obtidos foram competitivos com outros trabalhos da literatura, onde se provou a otimalidade em alguns casos e obteve-se melhores resultados e com melhor desempenho em outros. Em suma, as abordagens propostas permitem que o ferramental de outros problemas seja aplicado ao planejamento de redes móveis sem fio, além de apresentarem novos desafios científicos para a área.

\section{Introdução}

Os problemas de alocação de canais (channel assignment problems - CAP) constituem uma importante classe de problemas, tanto por questões teóricas quanto práticas. O objetivo dos mesmos consiste em atribuir porções do espectro eletromagnético, denominadas de canais, de acordo com as demandas da rede sem fio, de forma a minimizar ou eliminar possíveis interferências que degradam a qualidade do serviço prestado. $\mathrm{O}$ aumento da demanda por serviços de comunicação móvel tem tornado importante a pesquisa envolvendo este problema. Um dos primeiros trabalhos com a visão de otimização combinatória foi 
publicado em 1980 [Hale 1980] e desde então esta abordagem tem sido amplamente difundida [Dias 2014, Koster 1999, Audhya et al. 2011].

Além da importância prática, tal cenário é uma importante fonte de pesquisa científica. Variações de problemas clássicos de otimização combinatória são usadas para modelagem do CAP, como a classe de problemas de colorações de vértices em grafos, onde podem ser aplicados métodos exatos e aproximados para a resolução cada vez mais eficiente dos mesmos. Tal correlação também permite que um enfoque teórico dos problemas seja explorado, o que ainda é pouco presente em trabalhos relacionados.

Sendo assim, o principal objetivo da dissertação é fornecer contribuições científicas para a resolução mais eficiente do problema da alocação de canais em redes móveis sem fio em diferentes cenários de aplicação, com ênfase no Problema da Minimização do Maior Canal Alocado - Min-Max Channel Assignment Problem (Min-Max CAP), de tal forma a adaptar e propor novos modelos teóricos de problemas computacionais clássicos, estender e aplicar formulações matemáticas. bem como propor estratégias algorítmicas aplicando o ferramental da áreas de Otimização Combinatória, envolvendo Algoritmos, Teoria dos Grafos, Programação Inteira e por Restrições, bem como Geometria de Distâncias [Dias 2014, Freitas et al. 2014]. ${ }^{1}$

\section{Modelos teóricos propostos}

Por meio do estudo da literatura do problema de alocação de canais e suas características, além do ferramental de Otimização Combinatória, foi possível identificar modelos teóricos para a representação dos cenários do problema.

O primeiro modelo proposto foi feito utilizando colorações de vértices em grafos para representação do problema, de tal forma que cada estação da rede corresponde a um vértice do grafo, as interferências entre estações são representadas por meio de arestas ponderadas e os canais equivalem às cores que serão usadas pelos vértices. No entanto, com base em características adicionais que estão presentes na alocação de canais, foi possível identificar modelos baseados em colorações restritas existentes, tais como multicoloração, lista-coloração e $(\gamma, \mu)$-coloração, resultando no modelo proposto de coloração em grafos $(\boldsymbol{\gamma}, \boldsymbol{\mu})$-channel-multicoloring [Dias et al. 2013a]. Um exemplo pode ser visto na Figura 1.

O segundo modelo proposto foi desenvolvido com base em teoria de escalonamento, uma vez que, na literatura, já existia a correlação entre essa classe de problemas e coloração em grafos, mas não uma associação direta com alocação de canais. Para isso, os canais foram considerados como os instantes de tempo do escalonamento e cada chamada como uma tarefa de tempo unitário, com restrições de janela de tempo e tempos de preparação (setup) dependentes da sequência, com o objetivo de minimizar o maior tempo de completude dentre todas as tarefas (o makespan). O modelo proposto de escalonamento em máquinas paralelas, então, é definido na notação clássica de três campos por $\left(\boldsymbol{P}\left|\boldsymbol{p}_{\boldsymbol{j}}=\mathbf{1}, \boldsymbol{r}_{\boldsymbol{j}}, \overline{\boldsymbol{d}_{\boldsymbol{j}}}, \boldsymbol{s}_{\boldsymbol{j} \boldsymbol{m}}\right| \boldsymbol{C}_{\boldsymbol{m a x}}\right.$ [Dias et al. 2012]. Um exemplo pode ser visto na Figura 1.

O terceiro modelo proposto foi feito com base na observação de que as restrições

\footnotetext{
${ }^{1}$ Conteúdo abordado disponível em http://algox.icomp.ufam.edu.br/index.php/ channel-assignment.
} 


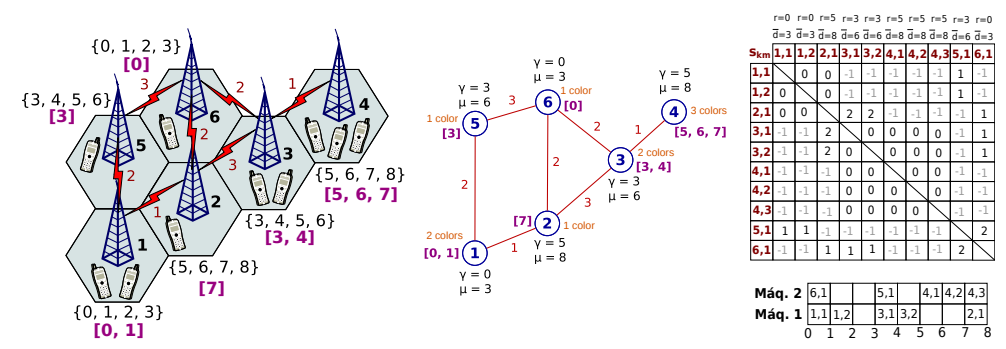

Figura 1. Exemplo de alocação de canais e seus modelos em coloração de grafos e escalonamento de tarefas.

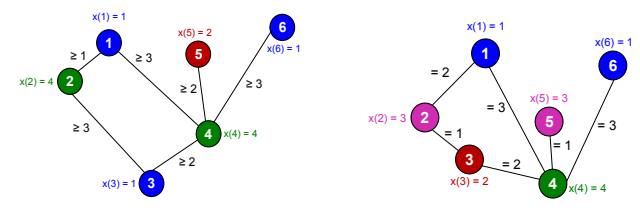

Figura 2. Exemplos de colorações com distâncias.

de separações entre canais consistem em um tipo de distância, o que possibilita a utilização de geometria de distâncias para modelagem do problema. Partindo do modelo de coloração em grafos para a alocação de canais e de modelos de geometria de distâncias para modelagem molecular, cada vértice deve ser projetado na reta $\left(\mathbb{R}^{1}\right)$, considerando apenas os números naturais da mesma, que serão pontos de cores a serem atribuídos a cada vértice. Devido à projeção ser apenas em uma dimensão, a norma é dada pela diferença absoluta entre os pontos de cores de cada vértice, o que permite a validação das restrições de separação da mesma forma que ocorre com os outros modelos. [Freitas et al. 2014]. Exemplos são dados na Figura 2.

Formulações de programação inteira e por restrições foram propostas com base nas características dos modelos definidos acima. As definições e conceitos relacionados a tais modelos pode ser visto no Capítulo 4 da dissertação.

\section{Estratégias algorítmicas propostas}

Para a resolução das variações do problema tratadas neste trabalho, foram implementadas estratégias algorítmicas exatas. bem como heurísticas fortemente baseadas em busca local.

O primeiro método proposto foi uma meta-heurística simulated annealing. Para a alocação de canais, uma estrutura de solução foi codificada, com base no modelo de escalonamento proposto, como um vetor de pares $(i, k)$, onde $i$ é uma estação e $k$ indica que o par refere-se a $k$-ésima chamada de $i$. Para a determinação da alocação em si, é utilizada a regra de alocação exaustiva de frequência (frequency exhaustive allocation - FEA), onde, seguindo a ordem das chamadas no vetor, é fornecido o menor canal para cada chamada tal que o mesmo não cause interferência com as chamadas que já receberam canais. A vizinhança utilizada no processo de determinação de uma nova solução é baseada em trocas entre pares, onde, no vetor de chamadas, são selecionadas duas posições tais que as estações sejam diferentes (isto é, dois pares $(i, k)$ e $(j, m)$ tais que $i \neq j$ ) e os pares das mesmas trocam de posição entre si. As posições utilizadas são selecionadas aleatoriamente. A cada nova solução, se a mesma tiver custo menor que a solução corrente atual, 
a nova passa a ser a corrente, caso contrário, com probabilidade $e^{(-\Delta / t)}$, a substituição é feita (onde $\Delta$ é a diferença entre custos e $t$ é a temperatura atual). São geradas novas soluções até que o equilíbrio termodinâmico seja atingido (no caso deste trabalho, número de soluções geradas), no qual a temperatura é decrementada.

Ao atingir a temperatura final, o algoritmo é encerrado e a melhor solução obtida durante todo o processo é retornada. Tal vizinhança também foi utilizada para o desenvolvimento de uma busca local iterada (iterated local search - ILS), porém, devido ao número de chamadas ser grande, a exploração total da vizinhança se mostrou muito cara.

Outro método utilizado foi a utilização dos algoritmos de branch-and-cut da ferramenta IBM/ILOG CPLEX a um modelo de programação inteira do problema de alocação de canais baseado no modelo de coloração em grafos. Para melhor eficiência da ferramenta, o modelo foi alimentado pelas soluções obtidas em execuções do simulated annealing. Ambas as abordagens foram aplicadas nas instâncias de benchmark Philadelphia, Helsinki e um conjunto artificial de 55 estações, sendo possível a obtenção, com o simulated annealing desenvolvido, de melhores resultados que os reportados na literatura durante a elaboração da dissertação, além das soluções ótimas para tais instâncias por meio do CPLEX, que ainda não haviam sido reportadas.

Uma terceira estratégia foi desenvolvida envolvendo os modelos de geometria de distância propostos. Com base no algoritmo branch-and-prune para o problema de modelagem molecular, o método branch-prune-and-bound foi desenvolvido, onde são feitas verificações de factibilidade a cada vértice colorido (prunings) e de qualidade, de forma que soluções parciais que não serão melhores que a solução incumbente atual serão descartadas da enumeração (boundings).

Três versões do método foram implementadas: a primeira, chamada de BPB-Prev, inicia atribuindo a cor 1 a um vértice e, em seguida, atribui cores a vértices vizinhos, respeitando a distância imposta na aresta (isto é, sendo $i$ o último vértice colorido, o próximo vértice $j$ receberá uma cor igual a soma da cor de $i$ com o peso da aresta entre $i$ e $j$ ). Quando uma cor atribuída a um vértice viola uma distância (uma vez que, na determinação da cor, é levada em conta apenas uma aresta incidente e não todas), é feita uma operação de pruning e, se a cor usada for maior que o custo atual da solução parcial, é feita uma operação de bounding. A segunda versão, chamada de BPB-Select, leva em conta todas as arestas na determinação da cor, resolvendo um sistema de equações e inequações de valores absolutos com base nos vértices já coloridos e nas distâncias das arestas, efetuando prunings e boundings da mesma forma. A terceira versão é utilizada em cenários com listas de cores (ou seja, domínios de frequência), de forma que toda vez que um vértice é colorido, as cores das listas dos vizinhos que violem a distância da aresta entre eles são bloqueadas. Se alguma lista se tornar vazia, a solução parcial é inválida e pode ser descartada. Tal procedimento é similar a algoritmos de propagação utilizados em programação por restrições, o que motivou também a implementação posterior de um modelo de programação por restrições utilizando a ferramenta CP Optimizer, parte do CPLEX, para resolução dos problemas.

A descrição completa dos algoritmos pode ser vista no Capítulo 5 da dissertação, e resultados númericos obtidos em experimentos computacionais são detalhados no Capítulo 6. 


\section{Prêmios e publicações}

Com os resultados deste trabalho, foram feitas diversas publicações científicas em eventos e revistas, tendo algumas sido premiadas como alguns dos melhores trabalhos dos veículos correspondentes. As principais publicações já disponíveis são citadas a seguir.

1. "Distance Geometry Approach for Special Graph Coloring Problems", a ser publicado no periódico International Transactions on Operational Research, editado pela International Federation of Operational Research Societies que reúne as principais sociedades de pesquisa operacional do mundo [Dias et al. 2015].

2. "Algorithms for min-max channel assignment problems in wireless networks", submetido ao periódico Computers and Operations Research (Qualis A1) [Dias et al. 2014].

3. "On feasibility conditions in graph coloring problems with distance constraints", [Freitas et al. 2014], apresentado no Workshop on Many Faces of Distances, realizado de 22 a 24 de outubro de 2014 em Campinas - SP. O trabalho foi selecionado como um dos quatro melhores apresentados no evento, que contou com a comunidade científica mundial de geometria de distâncias.

4. "On graph coloring problems with distance constraints" [Dias et al. 2013b], publicado no Workshop on Distance Geometry and Applications (DGA 2013), realizado de 24 a 27 de junho de 2013 em Manaus - AM, e premiado como um dos cinco melhores do evento e apresentado na forma oral e de pôster. $\mathrm{O}$ evento reuniu pesquisadores da área de geometria de distâncias, os quais selecionaram os melhores trabalhos para exposição oral, além de uma sessão de pôsteres.

5. "A hybrid genetic algorithm with local search approach for E/T scheduling problems on identical parallel machines" [Amorim et al. 2013] (co-autor), trabalho apresentado a 15th Annual Conference Companion on Genetic and Evolutionary Computation (GECCO 2013 - evento de Qualis A1), realizado de 06 a 10 de julho de 2015 em Amsterdã - Holanda.

6. "Simulated annealing para a alocação de canais em redes móveis celulares" [Dias et al. 2013a], publicado no XLV Simpósio Brasileiro de Pesquisa Operacional (SBPO 2013), realizado de 16 e 19 de setembro de 2013 em Natal - RN. O SBPO é o principal fórum científico brasileiro da área de pesquisa operacional, otimização e suas aplicações.

7. "Alocação de canais em redes celulares sem fio: algoritmos e modelos teóricos em grafos e escalonamento" [Dias et al. 2012], publicado no XVI Congreso Latino-Iberoamericano de Investigación Operativa, realizado em conjunto com o XLIV Simpósio Brasileiro de Pesquisa Operacional (CLAIO-SBPO 2012) de 24 e 28 de setembro de 2012 no Rio de Janeiro - RJ. O trabalho foi escolhido como um dos quatro melhores submetidos para o Prêmio de Iniciação Científica do evento e selecionado para apresentação oral.

Resultados parciais obtidos durante a pesquisa também foram apresentados em importantes conferências de Otimização Combinatória e Pesquisa Operacional, tais como IFORS 2014, INFORMS 2014, LAWCG 2014, EURO 2012/2013/2014 e OR 2013.

\section{Contribuições da pesquisa e considerações finais}

A primeira contribuição direta do trabalho consiste em um levantamento bibliográfico da literatura de alocação de canais, sendo que, além da exploração dos esquemas utilizados em tecnologias atuais, tais como GSM, CDMA, 3G e LTE, foi realizado um estudo da alocação de canais em redes de rádios cognitivos, que consistem em uma alternativa para melhor utilização do espectro eletromagnético, uma vez que fazem um uso oportunista de faixas de frequência subutilizadas, sempre garantindo a prioridade dos usuários 
licenciados. O trabalho também apresenta uma proposta de uso dos resultados para redes celulares na alocação dinâmica em redes cognitivas.

Os modelos teóricos propostos neste trabalho possibilitaram a expansão do conhecimento envolvendo correlações entre problemas clássicos de Otimização Combinatória, além de uma visão aplicada a cenários reais, sendo, neste caso, envolvendo redes de comunicação móvel. Os modelos de coloração em grafos expandiram uma abordagem tradicional na literatura de alocação de canais, enquanto os modelos de escalonamento de tarefas abrem a possibilidade da utilização de todo o ferramental disponível para essa classe de problemas, cuja pesquisa científica é bastante ampla, no planejamento das redes móveis.

A utilização de conceitos de geometria de distâncias e teoria dos grafos também permitiu que novos problemas fossem identificados, como colorações com restrições de igualdade entre vértices, onde, posteriormente à dissertação, estudos de factibilidade e complexidade foram realizados, aumentando o conhecimento teórico da área e possibilitando novas aplicações.

Por fim, foi possível obter melhores soluções, incluindo resultados de otimalidade para instâncias clássicas da literatura, o que contribuirá para melhores comparações de algoritmos posteriores. Como contribuição adicional, foram gerados conjuntos adicionais de instâncias mais difíceis, onde foi possível verificar o comportamento tanto dos algoritmos quanto dos modelos propostos, o que apresenta um desafio adicional a ser tratado em trabalhos futuros.

\section{Referências}

Amorim, R., Dias, B., Freitas, R., and Uchoa, E. (2013). A hybrid genetic algorithm with local search approach for e/t scheduling problems on identical parallel machines. In Proceedings of 15th Annual Conference on Genetic and Evolutionary Computation. ACM.

Audhya, G., Sinha, K., Ghosh, S., and Sinha, B. (2011). A survey on the channel assignment problem in wireless networks. Wireless Communications and Mobile Computing, 11:583-609.

Dias, B. (2014). Modelos teóricos e algoritmos para a otimização da alocação de canais em redes móveis sem fio. Master's thesis, Universidade Federal do Amazonas, Manaus - AM, Brasil.

Dias, B., Freitas, R., and Maculan, N. (2012). Alocação de canais em redes celulares sem fio: algoritmos e modelos teóricos em grafos e escalonamento. In Anais do XLIV Simpósio Brasileiro de Pesquisa Operacional. Sociedade Brasileira de Pesquisa Operacional (SOBRAPO).

Dias, B., Freitas, R., and Maculan, N. (2013a). Simulated annealing para a alocação de canais em redes móveis celulares. In Anais do XLV Simpósio Brasileiro de Pesquisa Operacional. Sociedade Brasileira de Pesquisa Operacional (SOBRAPO).

Dias, B., Freitas, R., and Maculan, N. (2014). Algorithms for min-max channel assignment problems in wireless networks. Computers and Operations Research. Submetido.

Dias, B., Freitas, R., Maculan, N., Szwarcfiter, J., and Michelon, P. (2015). Distance geometry approach for special graph coloring problems. International Transactions on Operational Research. A ser publicado.

Dias, B., Freitas, R., and Szwarcfiter, J. (2013b). On graph coloring problems with distance constraints. In Proceedings of I Workshop on Distance Geometry and Applications (DGA 2013).

Freitas, R., Dias, B., Maculan, N., and Szwarcfiter, J. (2014). On feasibility conditions in graph coloring problems with distance constraints. In Many Faces of Distances (Workshop).

Hale, W. K. (1980). Frequency assignment: Theory and applications. Proceedings of the IEEE, 25:14971514.

Koster, A. (1999). Frequency assignment: models and algorithms. Universiteit Maastricht. 